\title{
Influence du stade de maturité de la plante de maïs récoltée pour ensilage sur la cinétique de dégradation de l'ensilage et de quelques autres aliments dans le rumen
}

\author{
A Mayombo, I Dufrasne, L Istasse, M Gielen, N Korsak, JM Bienfait \\ Service de Nutrition, faculté de Médecine vétérinaire, université de Liège B 43, Sart Tilman, \\ 4000 Liège, Belgique
}

\begin{abstract}
Summary - Effects of whole plant maize silage harvest date on kinetics of disappearance of the silage components and of some other feedstuffs. Three maize silages differing in stage of maturity (cut 1: $25.1 \%$ dry matter (DM); cut 2: $29.8 \% \mathrm{DM}$ and cut 3: $32.3 \%$ ) were offered to 6 rumen-fistulated bulls. For 10 different feedstuffs compared, except for barley, DM disappearance in the rumen measured in sacco at time to and for the short incubation times was higher when cut 1 was offered. DM disappearance of grain cut 1 was higher than that of the 2 other cuts; opposite findings were observed for the stalks and leaves. The concentration of volatile fatty acids in the rumen fluid was higher in cut 1.
\end{abstract}

L'ensilage de maïs (EM) est fréquemment utilisé comme aliment de base dans l'engraissement du taurillon. Les différents composants de l'ensilage (tiges, feuilles, grains) sont dégradés par les microorganismes du rumen. La date de récolte de l'ensilage est un des critères qui déterminent la valeur nutritive de l'aliment. L'incubation de sachets de nylon dans le rumen permet de suivre la cinétique de dégradation des aliments au cours du temps.

Matériel et méthodes - La cinétique de dégradation dans le rumen et les fermentations ont été étudiées sur 6 taureaux munis d'une canule du rumen, soit 2 animaux par type d'EM. Ces animaux recevaient une ration à base d'EM à 25,1 (coupe $1: \mathrm{C} 1$ ), à 29,8 (coupe $2: \mathrm{C} 2$ ) ou $32,3 \%$ (coupe $3: \mathrm{C} 3$ ) de matière sèche (MS). L'EM représentait $60 \%$ des apports en MS; la ration était complémentée par du tourteau de soja $(1,55 \mathrm{~kg} / \mathrm{j})$ et des pulpes séchées. Les quantités d'aliments distribués étaient proches d' "ad libitum". La dégradabilité dans le rumen a été déterminée sur 10 aliments de composition chimique très variable : des grains et des tiges + feuilles des ensilages $\mathrm{C} 1, \mathrm{C} 2$ et $\mathrm{C} 3$; du tourteau de soja (TS), des pulpes séchées (PS), de l'orge $(O)$, du maïs grain sec (MG). Les échantillons des fractions d'ensilage ont été obtenus par séparation manuelle et séchés dans une étuve à $50^{\circ} \mathrm{C}$. Tous les aliments ont été broyés dans un broyeur à marteaux muni d'une grille de $10 \mathrm{~mm}$. La dégradabilité a été déterminée par la technique des sachets de nylon décrite par Mehrez et Orskov (1977). Les sachets ont été incubés pendant $4,8,16,24$ ou $48 \mathrm{~h}$ dans le rumen et les mesures ont été répétées. Du liquide de rumen a été prélevé à intervalle de $2 \mathrm{~h}$ pendant une période de $14 \mathrm{~h}$ commençant avant le repas du matin, en vue du dosage des acides gras volatils (AGV), de l'ammoniac et du glucose.

Résultats et discussion - Toute ration confondue, la dégradation de la MS des grains de l'ensilage $C 1$ à $71,4 \%$ a été supérieure à celle des grains des 2 autres ensilages $(67,9$ et $67,6 \%$ ) (tableau I). Ces derniers ont été néanmoins plus dégradables que les grains de maiis $\sec (37,2 \%)$. Ces différences seraient à mettre en rapport avec le procédé de conservation et avec la structure macroscopique du grain d'amidon, qui évolue avec le stade de maturité. Paradoxalement, la situation a été inversée pour les tiges et les feuilles. Cette inversion pourrait être due au phénomène de caramélisation lors du séchage à l'étuve.

Pour tous les aliments, à l'exception de l'orge, la dégradation au temps to (coeffi- 
cient a, tableau I) et celle correspondant aux courtes périodes d'incubation de 2 et 4 $h$ ont été supérieures lorsque la ration contenait l'ensilage $\mathrm{C} 1$; cette situation correspondrait à un temps de latence plus faible. Pour les temps d'incubation plus longs, la dégradation a été supérieure avec la ration contenant l'ensilage C3. Certaines différences entre dégradabilité ont été significatives au seuil $P<0,05$ suivant les temps d'incubation et les aliments étudiés.

L'évolution en fonction du temps de la concentration en AGV a été classique. C'est avec la ration contenant l'ensilage $C 1$ que la concentration en AGV a été la plus élevée $(118,0,97,6$ et $101,5 \mathrm{mmol} / \mathrm{l}$ pour C1, C2 et $\mathrm{C} 3$ respectivement $(P<0,05$ ou $0,01)$. En revanche, il n'y a pas eu de diffé- rences significatives pour les concentrations en NH3 $(96,1,76,0$ et $80 \mathrm{mg} \mathrm{N} / \mathrm{l}$ pour $\mathrm{C} 1, \mathrm{C} 2, \mathrm{C} 3)$ et en glucose $(1046,0,721,2$ et $906,4 \mathrm{mg} / \mathrm{l})$.

En conclusion, les concentrations en AGV obtenues avec la ration contenant l'ensilage $\mathrm{C} 1$ corroborent les résultats d'intensité de la dégradation des grains, indiquant une bonne activité microbienne dans le rumen.

Remerciements - Travail réalisé avec la collaboration financière de l'IRSIA (Institut de recherches scientifiques dans l'industrie et l'agriculture).

Mehrez AZ, Orskov ER (1977) J Agric Sci (Camb) 88, 645-650

Tableau I. Influence du stade de maturité de l'EM (C1, C2 ou C3) sur la dégradation dans le rumen des 10 aliments étudiés.

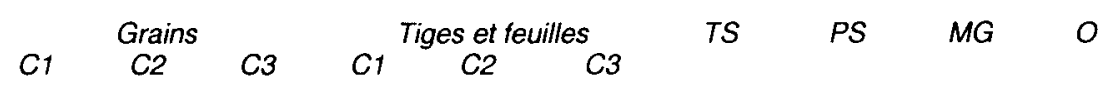

Toute ration confondue

$\begin{array}{lllllllllll}\text { a } & 40,8 & 35,4 & 36,1 & 19,8 & 29,1 & 30,9 & 15,6 & 0,8 & 6,9 & 34,5 \\ \text { b }^{2} & 58,0 & 64,0 & 61,1 & 49,9 & 43,3 & 55,8 & 84,4 & 93,0 & 93,1 & 51,8 \\ \text { C }^{3} & 0,067 & 0,062 & 0,064 & 0,043 & 0,045 & 0,028 & 0,070 & 0,083 & 0,029 & 0,165 \\ \text { DT } 4 & 71,4 & 67,9 & 67,6 & 40,6 & 47,7 & 48,7 & 61,1 & 54,8 & 37,2 & 72,5\end{array}$

Ration avec ensilage $\mathrm{C} 1$

$\begin{array}{lllllllllll}\text { a }^{1} & 51,9 & 47,7 & 46,9 & 26,6 & 34,1 & 32,4 & 18,4 & 11,1 & 9,0 & 24,4 \\ \text { b }^{2} & 48,1 & 52,4 & 53,1 & 63,4 & 42,8 & 50,6 & 81,6 & 83,8 & 91,0 & 59,7 \\ \text { C }^{3} & 0,050 & 0,049 & 0,043 & 0,021 & 0,030 & 0,028 & 0,066 & 0,069 & 0,026 & 0,249 \\ \text { D }^{4} & 73,8 & 71,3 & 69,1 & 43,0 & 48,4 & 48,5 & 61,1 & 55,9 & 36,5 & 72,5\end{array}$

Ration avec ensilage $\mathrm{C} 2$

$\begin{array}{lllllllllll}\text { a }^{1} & 26,9 & 26,9 & 21,8 & 18,9 & 24,6 & 29,5 & 9,7 & -2,1 & 7,1 & 22,7 \\ \text { b }^{2} & 69,4 & 73,2 & 70,8 & 49,0 & 46,5 & 43,5 & 90,3 & 96,4 & 92,9 & 63,9 \\ \text { C }^{3} & 0,080 & 0,054 & 0,083 & 0,052 & 0,048 & 0,044 & 0,073 & 0,078 & 0,026 & 0,150 \\ \text { D }^{4} & 66,6 & 61,6 & 62,9 & 41,7 & 45,3 & 47,9 & 59,1 & 52,4 & 35,2 & 68,3\end{array}$

Ration avec ensilage $\mathrm{C} 3$

\begin{tabular}{lllllllllll} 
a $^{1}$ & 41,8 & 27,7 & 37,5 & 11,9 & 28,9 & 28,8 & 18,0 & $-6,3$ & 4,0 & 45,6 \\
b $^{2}$ & 58,2 & 70,8 & 62,5 & 57,3 & 43,8 & 71,2 & 80,8 & 100,1 & 96,0 & 42,4 \\
c $^{3}$ & 0,076 & 0,094 & 0,068 & 0,089 & 0,053 & 0,024 & 0,075 & 0,099 & 0,036 & 0,148 \\
D $^{4}$ & 74,3 & 71,0 & 70,7 & 46,1 & 49,4 & 49,1 & 62,9 & 56,0 & 40,0 & 75,8 \\
\hline
\end{tabular}

1.2 .3 : constantes de l'équation exponentielle : $d=\mathrm{a}+\mathrm{b}\left(1-\mathrm{e}^{-c \mathfrak{l})} ;{ }^{4}\right.$ dégradabilité calculée avec un taux de vidange du rumen de $6 \% / \mathrm{h}$. 